\title{
Right ventricular pressure response to exercise in adults with isolated ventricular septal defect closed in early childhood
}

\begin{tabular}{|r|l|}
\hline Journal: & Cardiology in the Young \\
\hline Manuscript ID & CTY-17-Nov-422.R1 \\
\hline Manuscript Type: & Original Article \\
\hline Date Submitted by the Author: & n/a \\
\hline Complete List of Authors: & $\begin{array}{l}\text { Möller, Thomas; Oslo Universitetssykehus, Pediatric Cardiology } \\
\text { Lindberg, Harald; Oslo Universitetssykehus, Thoracic Surgery } \\
\text { Lund, May Brit; Oslo Universitetssykehus, Respiratory Medicine } \\
\text { Holmstrøm, Henrik; Rikshospitalet, Pediatric Cardiology } \\
\text { Døhlen, Gaute; Rikshospitalet, Pediatric Cardiology } \\
\text { Thaulow, Erik; Oslo Universitetssykehus, Pediatric Cardiology }\end{array}$ \\
\hline Keywords: & $\begin{array}{l}\text { congenital heart disease, ventricular septal defect, exercise capacity, } \\
\text { exercise-induced pulmonary arterial hypertension }\end{array}$ \\
\hline Manuscript Category: & Echocardiography and Scans \\
\hline \multicolumn{2}{|l}{} \\
\hline
\end{tabular}




\title{
Right ventricular pressure response to exercise in adults with isolated ventricular septal defect closed in early childhood.
}

\author{
Thomas Moller ${ }^{\mathrm{a}}$, Harald Lindberg ${ }^{\mathrm{b}}$, May Brit Lund ${ }^{\mathrm{c}}$, Henrik Holmstrom ${ }^{\mathrm{a}}$, Gaute \\ Dohlen $^{a}$, Erik Thaulow ${ }^{a}$ \\ ${ }^{a}$ Department of Paediatric Cardiology \\ ${ }^{b}$ Department of Thoracic Surgery \\ ${ }^{c}$ Department of Respiratory Medicine \\ Oslo University Hospital, Oslo, Norway
}

\section{Corresponding author:}

Thomas Möller, MD PhD

Department of Paediatric Cardiology, Division of Paediatric and Adolescent Medicine

Oslo University Hospital - Rikshospitalet

Postal address: P.O. Box 4950 Nydalen, 0424 Oslo, Norway

Phone: +47 90149702, Fax: +47 23072330

E-mail: thomas.moller@ous-hf.no / thofmol@gmail.com 


\begin{abstract}
We previously demonstrated an abnormally high right ventricular systolic pressure response to exercise in $50 \%$ of adolescents operated on for isolated ventricular septal defect. The present study investigated the prevalence of abnormal right ventricular systolic pressure response in 20 adult (age 30-45 years) patients who underwent surgery for early ventricular septal defect closure and its association with impaired ventricular function, pulmonary function, or exercise capacity. The patients underwent cardio-pulmonary tests, including exercise stress echocardiography.

Five of 19 patients (26\%) presented an abnormal right ventricular systolic pressure response to exercise $\geq 52 \mathrm{mmHg}$. Right ventricular systolic function was mixed, with normal tricuspid annular plane systolic excursion and fractional area change, but abnormal tricuspid annular systolic motion velocity (median $6.7 \mathrm{~cm} / \mathrm{s}$ ) and isovolumetric acceleration (median 0.8 $\mathrm{m} / \mathrm{sec}^{2}$ ). Left ventricular systolic and diastolic function was normal at rest as measured by the peak systolic velocity of the lateral wall and isovolumic acceleration, early diastolic velocity, and ratio of early diastolic flow to tissue velocity, except for ejection fraction (median 53\%). The myocardial performance index was abnormal for both the left and right ventricle. Peak oxygen uptake was normal (mean Z-score $-0.4,95 \%$ CI $-2.8-0.3$ ). There was no association between an abnormal right ventricular systolic pressure response during exercise and right or left ventricular function, pulmonary function, or exercise capacity.

Abnormal right ventricular pressure response is not more frequent in adult patients compared to adolescents. This does not support the theory of progressive pulmonary vascular disease following closure of left-to-right shunts.
\end{abstract}

Keywords: congenital heart disease, ventricular septal defect, exercise capacity, exerciseinduced pulmonary arterial hypertension 


\section{Introduction}

Isolated ventricular septal defect is the most common congenital heart disease (CHD)

1. Approximately one-third of symptomatic children with isolated ventricular septal defect and heart failure due to left-to-right shunt undergo surgical closure of the defect ${ }^{2}$. The risk of pulmonary vascular disease due to left-to-right shunting is one of the main arguments for early closure of the defect. The prevalence of pulmonary arterial hypertension in adult patients with a closed ventricular septal defect ranges from $3 \%$ to $13 \%$ in different studies ${ }^{3-5}$. We previously demonstrated an abnormal right ventricular systolic pressure response during exercise in $50 \%$ of adolescents (age 13 - 25 years) after surgical closure of an isolated ventricular septal defect ${ }^{6}$. A progressive pulmonary vascular disease was suggested. The aim of the present study was to define the prevalence of abnormal right ventricular systolic pressure response in adults operated on for ventricular septal defect early in life when investigated following the same protocol and tests as in our previous study ${ }^{6}$. We also investigated whether an abnormal right ventricular systolic pressure response to exercise is associated with impaired cardiac function, pulmonary function, or exercise capacity.

\section{Materials and methods}

\section{Patients}

We identified consecutive patients aged 30 to 45 years operated on at Oslo University Hospital (Rikshospitalet) for isolated ventricular septal defect before 5 years of age. Patient data were collected from the hospital's database of thoracic surgery, DATACOR ${ }^{7}$. Patients included in the study lived less than 4 hours travel distance from Oslo University Hospital and were able to perform an exercise test. We excluded patients with other known CHD at the time of the ventricular septal defect closure, and those with current unrelated medical conditions affecting heart or lung function, chromosomal abnormalities, or autoimmune 
diseases. Attention was paid to exclude patients with right ventricular outflow tract obstruction, as this condition interferes with the assessment of right ventricular function and non-invasive pressure estimation.

The study complies with the Declaration of Helsinki and was approved by the Norwegian Regional Committee for Medical Research Ethics. All participants provided written informed consent. The authors had full access to the data and take responsibility for its integrity. All authors have read and agreed to the manuscript as written.

\section{Measures}

The study participants underwent a full resting baseline echocardiographic assessment. Left ventricular systolic function was assessed by fractional shortening in M-mode, and biplane ejection fraction by Simpson's method from two-dimensional grayscale imaging ${ }^{8}$. We used pulsed wave tissue Doppler of the lateral mitral valve annulus to assess the peak systolic velocity and isovolumic acceleration ${ }^{9,10}$. Left ventricular diastolic function was assessed by peak early diastolic velocity and the ratio of early diastolic flow velocity through the mitral valve to the peak tissue velocity of the lateral annulus ${ }^{11,12}$. Right ventricular systolic function was measured using M-mode for the tricuspid annular plane systolic excursion ${ }^{13}$, and we measured right ventricular fractional area change from two-dimensional greyscale imaging ${ }^{14}$. We assessed the peak tricuspid annular plane systolic motion velocity and isovolumetric acceleration from pulsed wave tissue Doppler of the tricuspid annulus ${ }^{14}$. The combined systolic and diastolic function of either the left or right ventricle was assessed using the myocardial performance index, as measured with pulsed wave tissue Doppler from the basal segment of the free wall of the respective ventricle ${ }^{15,16}$. The right atrial pressure at rest was estimated by the vena cava inferior index ${ }^{17}$. All echocardiographic recordings were 
obtained with a Vivid E9 scanner (GE Healthcare, Horten, Norway). Echocardiographic studies were saved digitally (still frame and loops) for offline analysis.

The patients were examined with a cardiopulmonary exercise test on a treadmill ergometer with gas exchange analysis and electrocardiogram (VMax29, SensorMedics Corp., Yorba Linda, CA, USA) following a modified Bruce protocol ${ }^{18,19}$. Maximum oxygen uptake was expressed in milligrams per kilogram per minute, and the individual results were compared to reference values in healthy adults and expressed as a Z-score for the age-specific mean ${ }^{20}$. All tests were supervised by a cardiologist and performed until exhaustion. Early termination was determined according to common guidelines ${ }^{21}$ based on patient fatigue or the occurrence of predefined adverse events: arrhythmia, ischemia, chest pain, clinical signs of circulatory compromise, progressive fall in systolic blood pressure $>10 \%$, severe hypertension.

Two hours after treadmill testing, semi-supine cycle ergometry stress echocardiography (Ergoselect 1200 EL, Ergoline GmbH, Bitz, Germany) was performed as described previously ${ }^{22}$. A stepwise exercise protocol was used with a starting load of $25 \mathrm{~W}$, with a $25 \mathrm{~W}$ increase every third minute until $80 \%$ of the maximum heart rate from the cardiopulmonary exercise test was reached. Offline analysis of echocardiographic recordings was performed as described previously ${ }^{22}$. The maximal velocity of the tricuspid regurgitation jet was measured and averaged from as many image frames as possible during each workload stage. The right ventricular systolic pressure was calculated from each recording using the modified Bernoulli's equation, adding the right atrial pressure at rest to the calculated pressure gradient between right ventricle and right atrium ${ }^{23}$. Based on previous work by Grunig et al. ${ }^{24}$ and our own previous studies on the normal right ventricular systolic pressure response to exercise ${ }^{22}$, we defined a maximal right ventricular systolic pressure $\geq 52 \mathrm{mmHg}$ during exercise as abnormal. 
Pulmonary function tests included the measurement of static and dynamic lung volumes and gas diffusing capacity. All tests were performed according to the guidelines of the European Respiratory Society using the Vmax V6200 automated system (SensorMedics Corp., Yorba Linda, CA, USA). Total lung capacity, forced vital capacity, forced expiratory volume in one second, and gas diffusing capacity for carbon monoxide were recorded and reported in absolute values and as percent of predicted. The reference values recommended by the European Respiratory Society were used ${ }^{25}$. Whenever airway obstruction was suspected by the supervising pulmonologist, reversibility was tested by inhalation of a beta 2 receptor agonist.

\title{
Data analysis
}

Parametric data are presented as means with $95 \%$ confidence intervals $( \pm 2 \times$ standard error of the mean) and as medians with ranges. Unpaired student t-tests were performed to compare measurements under different conditions when the data were normally distributed. Pearson's correlation coefficient, linear regression, and logistic regression analysis were used to determine relationships between different variables. To determine functional predictors of the pathological pressure response to exercise, we computed four compound variables of cardiac and pulmonary function and performed a logistic regression analysis between all four variables and the presence or absence of a pathological pressure response. Compound variables were computed by averaging the standardized functional variables.

\author{
Statistical analysis \\ P-values $<0.05$ were considered significant. Statistical analysis was performed in \\ Microsoft Excel 2013 (Microsoft Corporation, Redmond WA, USA) and IBM SPSS Statistics \\ version 21 (IBM Corporation, New York NY, USA).
}




\section{Results \\ Patients}

We identified 57 consecutive patients operated on between 1972 and 1984 for isolated ventricular septal defect with a last registered residency within the target area. Of these patients, 13 had died: 6 deaths (10\%) before 2 years of age, 4 deaths (7\%) between 1 and 10 years of age, none between 10 and 20 years of age, 3 deaths (5\%) between 20 and 30 years of age, and none thereafter. Review of the medical charts of the survivors led to the exclusion of nine patients due to Down syndrome $(n=2)$, other known CHD $(n=3)$, or chronic muscular/skeletal issues precluding exercise testing $(n=2)$. Of the remaining 33 eligible patients, 13 declined to participate or failed to attend their study appointments. Therefore, we could include 20 patients (Table 1), 16 of which completed all parts of the examinations. Four patients did not perform the cardiopulmonary exercise test due to reasons unknown at the time of inclusion: technical failure, neurological impairment, recent muscular/skeletal injury, or anxiety. In addition, one patient who did not perform the cardiopulmonary exercise test also did not cooperate sufficiently during the pulmonary function test.

The median age at surgical closure of the ventricular septal defect was 44 months; a patch had been used in nine patients, direct suture in four patients, and documentation of the surgical technique was not available for the remaining seven patients. Two patients had been operated on for late diagnosed coarctation of the aorta with left thoracotomy several years after ventricular septal defect closure, without signs of recoarctation on follow-up. Both patients were normotensive without medication. One patient was diagnosed during the study visit with a borderline hypoplastic arch, which did not require intervention. One female patient had developed paroxysmal atrial fibrillation and was on permanent treatment with a beta-blocker and flecainide. Two patients had received permanent pacemaker treatment due to 
third degree atrioventricular block many years after the ventricular septal defect closure. Two patients had recently been through a medical investigation for palpitations or chest pain without pathological findings. One patient had been treated for mild bronchial asthma and presented no reversibility on the pulmonary function test.

\section{Findings}

Left ventricular systolic and global function expressed as the median left ventricular ejection fraction and myocardial performance index were slightly abnormal (Table 2). In contrast, the less load-dependent median peak systolic lateral mitral annulus velocity and median left ventricular isovolumetric acceleration were within normal limits. The left ventricular diastolic function expressed as peak early diastolic lateral mitral annulus velocity and the ratio of early diastolic mitral flow velocity and peak early diastolic lateral mitral annulus velocity was normal. Right ventricular systolic function at rest demonstrated divergent results. The median tricuspid annular plane systolic excursion and fractional area change were within normal limits, whereas the median tricuspid annular systolic motion velocity and median right ventricular isovolumetric acceleration were abnormal. The global right ventricular function measured by the median right ventricular myocardial performance index was also abnormal.

Electrocardiograms showed that two patients had a paced rhythm, with one in atrial fibrillation, and the remaining 17 patients were in sinus rhythm. Three patients had a right bundle branch block, but the remaining had no conduction disturbance.

The ratio of forced expiratory volume in one second and forced vital capacity were below the normal ranges (median $75 \%$ of predicted) (Table 3). Otherwise, lung volumes, dynamic parameters, and gas diffusing capacity covered broad ranges but were within normal limits ${ }^{25}$. There was no correlation between lung volumes, dynamic airway measurements, or 
diffusion lung capacity for carbon monoxide and the right ventricular systolic pressure response to exercise.

Cardiopulmonary exercise tests revealed normal exercise capacity. Compared to ageand gender-specific normal values, the average Z-score was - 0.4 (95\% confidence interval $2.8-0.3)$ for the whole group. The average peak oxygen uptake was $32.1 \mathrm{ml} / \mathrm{kg} / \mathrm{min}(95 \%$ confidence interval 25.7 - 38.6) for females and $36.8 \mathrm{ml} / \mathrm{kg} / \mathrm{min}(95 \%$ confidence interval $31.2-42.4$ ) for males. Due to the inclusion of two pacemaker-dependent individuals, we did not analyze the maximal heart rate during exercise.

Five of 19 patients $(26 \%)$ presented with an abnormal right ventricular systolic pressure response to exercise, defined as a maximal right ventricular systolic pressure $\geq 52$ mmHg during semi-supine cycle ergometry stress echocardiography (Table 4, Fig. 1). We found no difference in peak oxygen uptake or pulmonary function between patients with or without an abnormal right ventricular systolic pressure increase during exercise.

\section{Logistic regression}

Logistic regression analysis did not identify any predictive value for left ventricular systolic or diastolic function, right ventricular systolic function, or pulmonary function in terms of the odds ratio for an abnormal right ventricular systolic pressure response to exercise. Normal and abnormal responders did not differ in their exercise capacity or in relative changes in left ventricular (peak systolic lateral mitral annulus velocity, peak early diastolic lateral mitral annulus velocity, isovolumetric acceleration, myocardial performance index) or right ventricular (tricuspid annular plane systolic excursion, peak systolic lateral mitral annulus velocity, isovolumetric acceleration) functional parameters during exercise.

\section{Discussion}




\section{Abnormal right ventricular pressure response to exercise}

Our results demonstrate an abnormal right ventricular systolic pressure response to exercise in $26 \%$ of adults with a surgically closed ventricular septal defect, independent of ventricular function, pulmonary function, or exercise capacity. The prevalence of an abnormal right ventricular systolic pressure response to exercise was lower in our group of adult patients in their fourth and fifth decades than our previous study in adolescents aged 14-25 years ${ }^{6}$. Consequently, our data do not support the concern of a common progressive pulmonary vascular disease after defect closure early in life. Echocardiography did not show any indications of pulmonary arterial hypertension at rest, which should be expected in 4-13\% of cases according to the literature ${ }^{4,5}$.

Our own previous work in adolescents with isolated ventricular septal defects demonstrated normal right ventricular systolic pressure at rest but an abnormal increase in right ventricular systolic pressure during semi-supine cycle ergometry stress echocardiography when compared to a healthy age- and gender-matched control group ${ }^{6}$. An abnormal right ventricular systolic pressure response to exercise and, thus, exercise-induced pulmonary hypertension could theoretically represent an early stage of pulmonary vascular disease. The upper normal limits for the right ventricular systolic pressure response to exercise in healthy non-athletic adults measured non-invasively have been published previously ${ }^{22,24}$. Our data do not indicate a higher prevalence of exercise-induced pulmonary hypertension in an older ventricular septal defect population compared to previous results in adolescents. Therefore, a theoretically progressive character of pulmonary vascular changes late after defect closure is not supported by our data. However, in light of substantial preinclusion mortality and the enrollment of only 20 of 33 eligible patients, selection bias towards healthier individuals cannot be excluded. 


\section{Ventricular function}

As in adolescents, the pressure curves during incremental exercise continued to rise in individuals with an abnormal pressure response, whereas the curves of normal responders reached a plateau (Figure 1). However, the pressure phenomenon obviously does not separate the two groups in terms of ventricular myocardial reserve, and we were not able to demonstrate any relationship between the abnormal right ventricular systolic pressure response to exercise and systolic or diastolic right or left ventricular function at rest or during exercise. Hypothetically, the impaired right ventricular systolic function at rest is due to general defect-specific cardiac injury or surgical trauma rather than being a direct consequence of chronically increased afterload for the right ventricle due to subclinical pulmonary vascular disease and elevated pulmonary vascular resistance.

Echocardiography at rest confirmed normal or only mildly impaired left ventricular function, both systolic and diastolic, which was also reported by Roos-Hesselink et al. in adults late after surgical ventricular septal defect closure ${ }^{5}$. In contrast, right ventricular performance at rest was not consistently subnormal in our study population, at least partly confirming our previous findings of reduced right ventricular systolic function in adolescents after surgical ventricular septal defect closure ${ }^{6}$. A recent MRI study by Heiberg et al. showed structural and functional changes in the right ventricles of adults operated on for a ventricular septal defect very early in life ${ }^{26}$.

\section{Pulmonary function}

Impaired pulmonary function and its impact on mortality have been demonstrated for heterogenic groups of adults with congenital heart disease ${ }^{27-30}$. In our study group, pulmonary function in terms of dynamic parameters (ratio of forced expiratory volume in one second and forced vital capacity) was in the lower normal range, whereas lung volumes and gas diffusing 
capacity presented a wide range suggestive of a possible impact of repeated thoracotomy and neurological injury. In theory, an abnormal right ventricular systolic pressure response to exercise could represent exercise-induced pulmonary hypertension and early pulmonary vascular disease, which should reduce the gas diffusing capacity. However, our data do not support this theory. Pulmonary function appears to not be a major cause of impaired exercise capacity in our study group.

\section{Exercise capacity}

The aerobic exercise capacity as measured by peak oxygen uptake was normal relative to recent values from a healthy Norwegian population ${ }^{20}$, which contradicts previous studies involving patients with isolated ventricular septal defects ${ }^{31,32}$. Furthermore, our peak oxygen uptake results are better than those reported for ventricular septal defect patients in Kempny et al.'s diagnosis-specific reference data for adults with $\mathrm{CHD}^{33}$. Because previous reports of aerobic capacity in CHD have commonly omitted the age at defect closure, we must suspect that our inclusion criterion of early defect closure before 5 years of age may have diminished the exercise intolerance. Another explanation for the normal peak oxygen uptake in our patients may be selection bias due to reservations against study participation in patients with exercise intolerance.

\section{Late morbidity and mortality}

Our patients exhibited significant late morbidity, and even mortality, which is in line with recent outcome studies ${ }^{3,4,34,35}$. The electrophysiological late morbidity (pacemaker implantation for sinus node disease or high degree atrioventricular block, atrial fibrillation) is in line with other reports ${ }^{5,35}$. Gabriels et al. recently reported late occurrence of left ventricular outflow tract obstruction at the valvar level as a significant late complication ${ }^{35}$, 
whereas several patients in our study were diagnosed after defect closure with an obstruction in the aortic arch, with or without the need of intervention.

A substantial number of surgical patients from the dataset used for recruitment had died at an early age and after the early post-operative period. Even if mortality analysis was outside the scope of our study, this finding is worrying. Other authors previously confirmed the occurrence of sudden death in adults with a closed ventricular septal defect, representing a large group among patients with $\mathrm{CHD}^{5,36}$. Thus, the general perception of ventricular septal defects as benign and simple cardiac defects can be questioned and the common practice of terminating clinical follow-up a few years after defect closure may be inadequate ${ }^{37}$. However, data allowing risk stratification for cardiac events in these patients do not exist.

\section{Limitations}

Our study group was limited in size. However, being able to recruit from a surgical database covering all patients in the south-eastern region of Norway at the time of surgery, we consider it a representative sample without bias towards more severe cases. A majority of our participants were not undergoing congenital cardiac follow-up.

The study did not include a healthy control group. Thus, based on the present data, we are unable to judge age-dependent normality or abnormality of right ventricular pressure response during exercise compared to healthy controls. However, as significant worsening from adolescent age was not found. Therefore, we do not consider natural pressure increase by aging as a strong confounder.

In light of the substantial late mortality, there may be bias towards fewer changes in the pulmonary circulation in our group of surviving patients. 
In conclusion, our study demonstrated an abnormal right ventricular systolic pressure response to exercise in $26 \%$ of adults with surgically closed ventricular septal defects. The prevalence of an abnormal right ventricular systolic pressure response to exercise is comparable to previous data in adolescents with ventricular septal defects. The hypothesis of progressive pulmonary vascular disease after defect closure early in life is not supported by our data. The abnormal right ventricular systolic pressure response to exercise was not associated with impaired ventricular function at rest or during exercise, pulmonary function, or exercise capacity. 


\begin{abstract}
Acknowledgments
We want to thank Harald Weedon-Fekjær, Oslo Center for Biostatistics and

Epidemiology, Research Support Services, Oslo University Hospital, for his assistance with the statistical analysis.
\end{abstract}

\title{
Financial Support
}

This project was financed by a personal post-doctoral grant from EXTRA funds from the Norwegian Foundation for Health and Rehabilitation (grant no. 2009/2/0340).

\section{Conflicts of interest}

None.

\section{Ethical standards}

The authors assert that all procedures contributing to this work comply with the ethical standards of the relevant national guidelines on human experimentation in Norway and with the Helsinki Declaration of 1975, as revised in 2008, and has been approved by the regional committees for medical and health research, South-Eastern Norway. 


\section{References}

1. Marelli AJ, Mackie AS, Ionescu-Ittu R, Rahme E and Pilote L. Congenital heart disease in the general population: changing prevalence and age distribution. Circulation 2007; 115: 163172.

2. Gersony WM. Natural history and decision-making in patients with ventricular septal defect. Progress in Pediatric Cardiology 2001; 14: 125-132.

3. Duffels MG, Engelfriet PM, Berger RM et al. Pulmonary arterial hypertension in congenital heart disease: an epidemiologic perspective from a Dutch registry. International Journal of Cardiology 2007; 120: 198-204.

4. Engelfriet PM, Duffels MG, Moller T et al. Pulmonary arterial hypertension in adults born with a heart septal defect: the Euro Heart Survey on adult congenital heart disease. Heart 2007; 93: 682-687.

5. Roos-Hesselink JW, Meijboom FJ, Spitaels SE et al. Outcome of patients after surgical closure of ventricular septal defect at young age: longitudinal follow-up of 22-34 years.

European Heart Journal 2004; 25: 1057-1062.

6. Moller T, Brun H, Fredriksen PM et al. Right ventricular systolic pressure response during exercise in adolescents born with atrial or ventricular septal defect. Am J Cardiol 2010; 105: $1610-1616$.

7. Svennevig JL, Bech J, Karlsen H, Amlie E and Olsen A. [From a registry to a clinical information system. Development of the Datacor system at the surgery department A, Rikshospitalet]. Tidsskr Nor Laegeforen 1995; 115: 1057-1059.

8. Lang RM, Bierig M, Devereux RB et al. Recommendations for chamber quantification. Eur J Echocardiogr 2006; 7: 79-108.

9. Roche SL, Vogel M, Pitkanen $\mathrm{O}$ et al. Isovolumic acceleration at rest and during exercise in children normal values for the left ventricle and first noninvasive demonstration of exercise-induced force-frequency relationships. J Am Coll Cardiol 2011; 57: 1100-1107. 10. Ruan Q and Nagueh SF. Effect of age on left ventricular systolic function in humans: a study of systolic isovolumic acceleration rate. Exp Physiol 2005; 90: 527-534.

11. De Sutter J, De Backer J, Van de Veire N, Velghe A, De Buyzere M and Gillebert TC. Effects of age, gender, and left ventricular mass on septal mitral annulus velocity (E') and the ratio of transmitral early peak velocity to $E^{\prime}$ (E/E'). Am J Cardiol 2005; 95: 1020-1023.

12. Nagueh SF, Appleton CP, Gillebert TC et al. Recommendations for the evaluation of left ventricular diastolic function by echocardiography. Eur J Echocardiogr 2009; 10: 165-193. 13. Kaul S, Tei C, Hopkins JM and Shah PM. Assessment of right ventricular function using two-dimensional echocardiography. American Heart Journal 1984; 107: 526-531.

14. Rudski LG, Lai WW, Afilalo J et al. Guidelines for the echocardiographic assessment of the right heart in adults: a report from the American Society of Echocardiography endorsed by the European Association of Echocardiography, a registered branch of the European Society of Cardiology, and the Canadian Society of Echocardiography. J Am Soc Echocardiogr 2010; 23: 685-713; quiz 786-688.

15. Pellett AA, Tolar WG, Merwin DG and Kerut EK. The Tei index: methodology and disease state values. Echocardiography 2004; 21: 669-672.

16. Tei C, Ling LH, Hodge DO et al. New index of combined systolic and diastolic myocardial performance: a simple and reproducible measure of cardiac function--a study in normals and dilated cardiomyopathy. Journal of cardiology 1995; 26: 357-366.

17. Kircher BJ, Himelman RB and Schiller NB. Noninvasive estimation of right atrial pressure from the inspiratory collapse of the inferior vena cava. American Journal of Cardiology 1990; 66: 493-496. 
18. Fredriksen PM, Ingjer F, Nystad W and Thaulow E. Aerobic endurance testing of children and adolescents--a comparison of two treadmill-protocols. Scandinavian Journal of Medicine and Science in Sports 1998; 8: 203-207.

19. Fredriksen PM, Ingjer F and Thaulow E. [A protocol for testing aerobic capacity in children and adolescents with congenital heart defects]. TidsskrNor Laegeforen 1998; 118 : 2636-2639.

20. Edvardsen E, Scient C, Hansen BH, Holme IM, Dyrstad SM and Anderssen SA. Reference values for cardiorespiratory response and fitness on the treadmill in a 20- to 85year-old population. Chest 2013; 144: 241-248.

21. Paridon SM, Alpert BS, Boas SR et al. Clinical stress testing in the pediatric age group: a statement from the American Heart Association Council on Cardiovascular Disease in the Young, Committee on Atherosclerosis, Hypertension, and Obesity in Youth. Circulation 2006; 113: 1905-1920.

22. Moller T, Peersen K, Pettersen E, Thaulow E, Holmstrom H and Fredriksen PM. Noninvasive measurement of the response of right ventricular pressure to exercise, and its relation to aerobic capacity. Cardiol Young 2009; 19: 465-473.

23. Yock PG and Popp RL. Noninvasive estimation of right ventricular systolic pressure by Doppler ultrasound in patients with tricuspid regurgitation. Circulation 1984; 70: 657-662. 24. Grunig E, Weissmann S, Ehlken N et al. Stress Doppler echocardiography in relatives of patients with idiopathic and familial pulmonary arterial hypertension: results of a multicenter European analysis of pulmonary artery pressure response to exercise and hypoxia. Circulation 2009; 119: 1747-1757.

25. Quanjer PH, Tammeling GJ, Cotes JE, Pedersen OF, Peslin R and Yernault JC. Lung volumes and forced ventilatory flows. Report Working Party Standardization of Lung Function Tests, European Community for Steel and Coal. Official Statement of the European Respiratory Society. The European respiratory journal Supplement 1993; 16: 5-40.

26. Heiberg J, Ringgaard S, Schmidt MR, Redington A and Hjortdal VE. Structural and functional alterations of the right ventricle are common in adults operated for ventricular septal defect as toddlers. European heart journal cardiovascular Imaging 2015; 16: 483-489. 27. Opotowsky AR. Abnormal spirometry in congenital heart disease: where do we go from here? Circulation 2013; 127: 865-867.

28. Rigolin VH, Li JS, Hanson MW et al. Role of right ventricular and pulmonary functional abnormalities in limiting exercise capacity in adults with congenital heart disease. Am J Cardiol 1997; 80: 315-322.

29. Ginde S, Bartz PJ, Hill GD et al. Restrictive lung disease is an independent predictor of exercise intolerance in the adult with congenital heart disease. Congenit Heart Dis 2013; 8: 246-254.

30. Alonso-Gonzalez R, Borgia F, Diller GP et al. Abnormal lung function in adults with congenital heart disease: prevalence, relation to cardiac anatomy, and association with survival. Circulation 2013; 127: 882-890.

31. Diller GP, Dimopoulos K, Okonko D et al. Exercise intolerance in adult congenital heart disease: comparative severity, correlates, and prognostic implication. Circulation 2005; 112: 828-835.

32. Dimopoulos K, Diller GP, Piepoli MF and Gatzoulis MA. Exercise intolerance in adults with congenital heart disease. Cardiology Clinics 2006; 24: 641-660, vii. 33. Kempny A, Dimopoulos K, Uebing A et al. Reference values for exercise limitations among adults with congenital heart disease. Relation to activities of daily life--single centre experience and review of published data. Eur Heart J 2012; 33: 1386-1396. 
34. Engelfriet P, Boersma E, Oechslin E et al. The spectrum of adult congenital heart disease in Europe: morbidity and mortality in a 5 year follow-up period: The Euro Heart Survey on adult congenital heart disease. European Heart Journal 2005; 26: 2325-2333.

35. Gabriels C, De Backer J, Pasquet A et al. Long-Term Outcome of Patients with Perimembranous Ventricular Septal Defect: Results from the Belgian Registry on Adult Congenital Heart Disease. Cardiology 2017; 136: 147-155.

36. Meijboom F, Szatmari A, Utens E et al. Long-term follow-up after surgical closure of ventricular septal defect in infancy and childhood. Journal of the American College of Cardiology 1994; 24: 1358-1364.

37. Baumgartner H, Bonhoeffer P, De Groot NM et al. ESC Guidelines for the management of grown-up congenital heart disease (new version 2010). Eur Heart J 2010; 31: 2915-2957. 38. Chahal NS, Lim TK, Jain P, Chambers JC, Kooner JS and Senior R. Normative reference values for the tissue Doppler imaging parameters of left ventricular function: a populationbased study. Eur J Echocardiogr 2010; 11: 51-56. 


\section{Tables}

$\underline{\text { Abbreviations for tables and figures in alphabetical order }}$

$\mathrm{BMI}=$ body mass index

$\mathrm{BP}=$ blood pressure

$\mathrm{DLCO}=$ diffusion lung capacity for carbon monoxide

$\mathrm{E}=$ early diastolic mitral flow velocity

$\mathrm{e}^{\prime}=$ peak early diastolic lateral mitral annulus velocity

$\mathrm{FAC}=$ fractional area change

FEV1 $=$ forced expiratory volume in one second

$\mathrm{FVC}=$ forced vital capacity

IVA $=$ isovolumetric acceleration

$\mathrm{LV}=$ left ventricle

MPI = myocardial performance index

$\mathrm{RV}=$ right ventricle

RVSP $=$ right ventricular systolic pressure

$\mathrm{s}^{\prime}=$ peak systolic lateral mitral annulus velocity

TAPSE $=$ tricuspid annular plane systolic excursion

TASM $=$ tricuspid annular systolic motion velocity

TLC $=$ total lung capacity

$\mathrm{VSD}=$ ventricular septal defect 
$\underline{\text { Table 1: basic patient characteristics }(\mathrm{N}=20)}$

$$
\text { Number Median (range) }
$$

Female / male $\quad 12 / 8$
Age [years]
$40(32-44)$
BMI $\left[\mathrm{kg} / \mathrm{m}^{2}\right]$
$24.7(18.5-33.9)$
Age at VSD closure [months] $\quad 44(7-61)$
Oxygen saturation at rest [\%] $\quad 99(97-100)$
Systolic blood pressure [mmHg] $118(101-155)$
Diastolic blood pressure [mmHg] $75(68$ - 94) 
Table 2 echocardiographic functional data at rest $(\mathrm{N}=20)$. Red marking of median values out of normal range.

$$
\text { Median (range) Normal range Reference }
$$

\section{Left ventricular function}

$\underline{\text { Systolic }}$

Shortening fraction $[\%]$

Biplane ejection fraction [\%]

$53(42-63)$

$25-45$

$\mathrm{s}^{\prime}[\mathrm{cm} / \mathrm{sec}]$

$8(5-10)$

$\geq 55$

$>5.8$

38

IVA (from LV) [m/sec $\left.{ }^{2}\right]$

$0.8(0.3-1.3)$

$\geq 0.69$

9,10

$\underline{\text { Diastolic }}$

$\mathrm{e}^{\prime}[\mathrm{cm} / \mathrm{sec}]$

$14(11-18)$

$\geq 14$

11,12

$\mathrm{E} / \mathrm{e}^{\prime}$

$6.0(2.8-12.0) \leq 10$

11

Global

MPI (from LV)

$0.46(0.24-0.63) \leq 0.39$

15,16

\section{Right ventricular function}

Systolic

TAPSE [mm]

$17.5(12.0-24.0) \quad \geq 16$

14

FAC [\%]

$35.5(21-43)$

$\geq 35$

14

TASM $[\mathrm{cm} / \mathrm{sec}]$

$6.7(1.1-9.8)$

$\geq 10$

14

IVA (from RV) [m/ $\left./ \mathrm{sec}^{2}\right]$

$0.8(0.1-2.5)$

$\geq 2.2$

14

Global

MPI (from RV)

$0.59(0.36-0.86) \quad \leq 0.4$

14 
Table 3: pulmonary function at rest $(\mathrm{N}=19)$. Red marking of median value below $80 \%$ of predicted.

$\begin{array}{lll} & \text { Median } & \text { Range } \\ \text { TLC [Liter] } & 5.4 & 3.2-7.6 \\ \text { TLC [\% pred.] } & 87 & 51-116 \\ \text { FVC [Liter] } & 3.4 & 2.3-5.5 \\ \text { FVC [\% pred.] } & 88 & 51-118 \\ \text { FEV1 [Liter] } & 2.5 & 1.8-4.0 \\ \text { FEV1 [\% pred.] } & 85 & 48-112 \\ \text { FEV1/FVC [\% pred.] } & 75 & 60-85 \\ \text { DLCO [mmol*kPa }{ }^{-1} * \min ^{-1} \text { ] } & 8.2 & 5.3-11.5 \\ \text { DLCO [\% pred.] } & 89 & 67-98\end{array}$


Table 4: responses to exercise during semi-supine cycle ergometry stress echocardiography $(\mathrm{N}=19)$ as mean values with $95 \%$ confidence intervals

$\begin{array}{llll} & \text { Baseline } & \text { Exercise } & \text { Increase [\%] } \\ \text { Heart rate }[\mathrm{bpm}] & 75.5(68.9-82.2) & 145.8(141.3-150.3) & 99.2(82.5-116.1) \\ \text { Systolic BP }[\mathrm{mmHg}] & 116.0(110.3-121.7) & 187.6(171.5-203.8) & 61.6(51.0-72.1) \\ \text { Left ventricular response } & & & \\ \mathrm{s}^{\prime}[\mathrm{cm} / \mathrm{sec}] & 5.2(4.5-6.0) & 8.9(7.5-10.4) & 75.5(53.7-97.4) \\ \mathrm{e}^{\prime}[\mathrm{cm} / \mathrm{sec}] & 9.8(8.6-10.9) & 14.4(13.1-15.6) & 56.1(31.5-80.7) \\ \text { IVA (from LV) }\left[\mathrm{m} / \mathrm{s}^{2}\right] & 0.79(0.64-0.95) & 2.32(1.80-2.83) & 236.0(140.1-331.8) \\ \text { MPI (from LV) } & 0.47(0.42-0.51) & 0.52(0.47-0.57) & 13.3(8.0-18.5)\end{array}$

Right ventricular response

$\begin{array}{lccc}\text { RVSP* }[\mathrm{mmHg}] & 26.2(24.6-27.7) & 43.1(37.5-48.7) & 65.5(46.3-84.7) \\ \text { TAPSE }[\mathrm{mm}] & 17.2(15.9-18.6) & 21.3(18.4-24.1) & 26.3(14.3-38.2) \\ \text { TASM }[\mathrm{cm} / \mathrm{sec}] & 5.8(4.5-7.0) & 9.4(8.0-10.7) & 100.5(49.7-151.4) \\ \text { IVA (from RV) }\left[\mathrm{m} / \mathrm{s}^{2}\right] & 0.77(0.51-1.03) & 1.68(1.22-2.14) & 219.2(112.5-325.8) \\ \text { * Upper normal limit of right ventricular systolic pressure during maximal exercise }<52 \\ \text { mmHg }\end{array}$




\section{Figure legends}

\section{Figure 1:}

Scatterplots of serial measurements of RVSP during exercise. Color code depending on normal non-responders $=$ black $(\mathrm{RVSP}<52 \mathrm{mmHg})$ or abnormal responders $=$ red $(\mathrm{RVSP} \geq$ $52 \mathrm{mmHg}$ ) by means of maximal RVSP during exercise. 


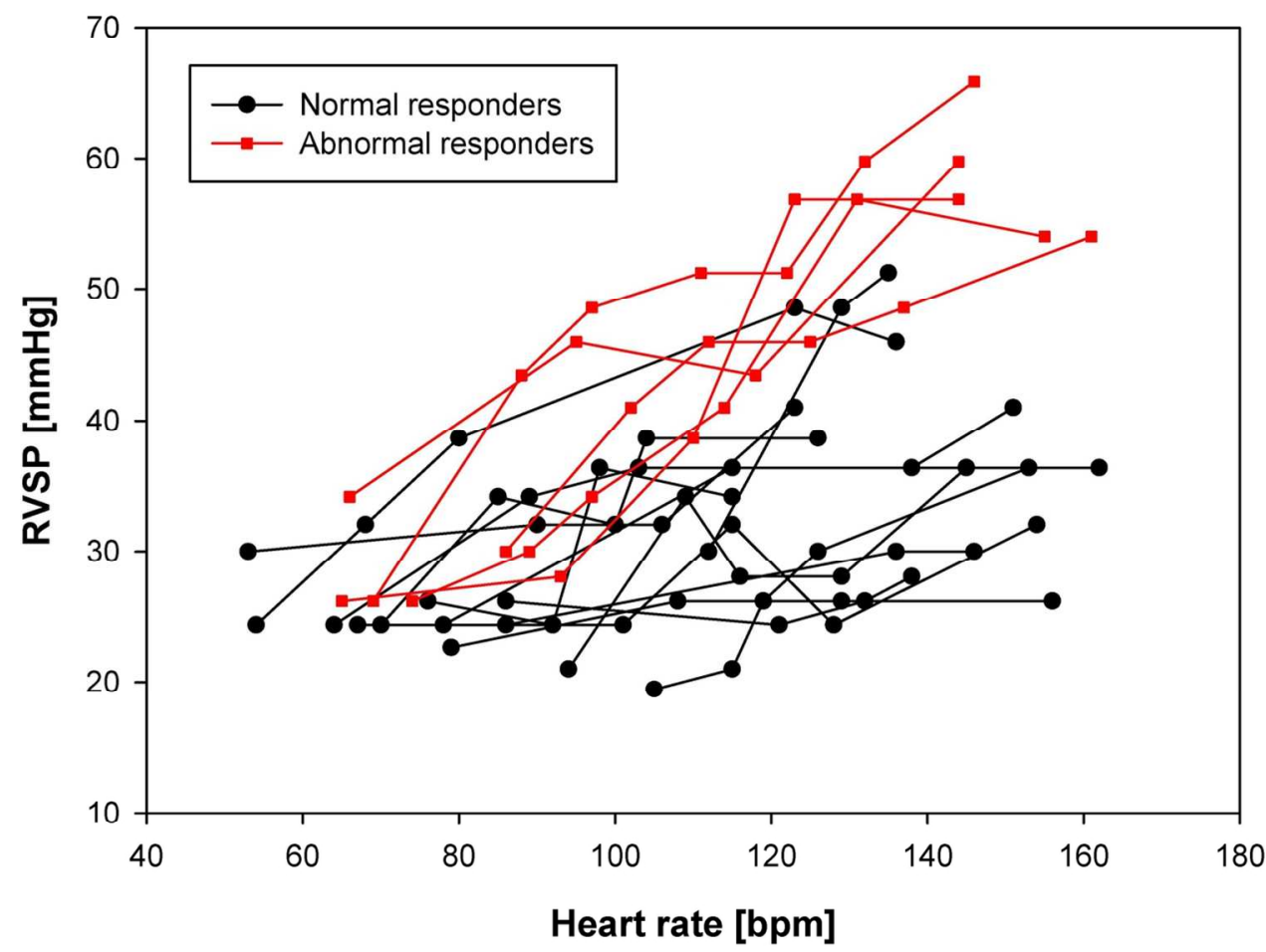

Scatterplots of serial measurements of RVSP during exercise. Color code depending on normal nonresponders $=$ black $(R V S P<52 \mathrm{mmHg}$ ) or abnormal responders $=$ red (RVSP $\geq 52 \mathrm{mmHg}$ ) by means of maximal RVSP during exercise.

$119 \times 96 \mathrm{~mm}(300 \times 300$ DPI) 